\title{
Regeneração de espécies arbóreas sob a influência de Merostachys multiramea Hack. (Poaceae) em uma floresta subtropical
}

\author{
Suzana Cyrino dos Santos ${ }^{1}$, Jean Carlos Budke ${ }^{1,3}$ e Adelcio Muller ${ }^{2}$
}

Recebido em 1/02/2011. Aceito em 8/02/2012

\begin{abstract}
RESUMO
(Regeneração de espécies arbóreas sob a influência de Merostachys multiramea Hack. (Poaceae) em uma floresta subtropical). A ocorrência de bambúseas é comum em muitas fisionomias florestais, sendo que a elevada abundância destas espécies pode interagir com a regeneração de espécies arbóreas. Foi avaliada a regeneração de espécies arbóreas e de Merostachys multiramea Hack. em uma área de transição entre Floresta Ombrófila Mista e Floresta Estacional no Sul do Brasil e comparada a estrutura de regeneração da área entre dois e quatro anos após floração, frutificação e morte de M. multiramea. Foram alocadas 25 unidades amostrais de $100 \mathrm{~m}^{2}$ dispostas de forma aleatória, onde foram amostrados todos os colmos vivos de $M$. multiramea e indivíduos regenerantes de espécies arbóreas $\geq 30 \mathrm{~cm}$ de altura e com perímetro à altura do peito $\leq 15 \mathrm{~cm}$. Foram amostrados 4.372 indivíduos vivos de bambu e 2.918 indivíduos regenerantes, pertencentes a 32 famílias e 75 espécies. A maioria das espécies é zoocórica, dependentes de luz para germinação e formadoras de dossel, porém, a abundância de indivíduos revelou maior contribuição de indivíduos autocóricos, tolerantes à sombra e de sub-bosque. A densidade de $M$. multiramea elevou-se ao longo dos quatro anos após período reprodutivo $(\mathrm{p}<0,05)$, com aumento da altura média dos colmos $(\mathrm{p}<0,01)$, mas sem alteração no número médio de colmos $(\mathrm{p}=0,46)$. Uma NPMANOVA revelou diferenças significativas entre composição e respectivas abundâncias entre os levantamentos, com maior similaridade entre as amostras de dois anos após o período reprodutivo de M. multiramea. Houve aumento da riqueza específica ao longo do tempo, estando diretamente associada à abundância de $M$. multiramea $(\mathrm{p}=0,002)$, porém, com diminuição da uniformidade de abundâncias entre espécies em regeneração.
\end{abstract}

Palavras-chave: dinâmica de mosaicos, estimadores de riqueza e diversidade, ecologia de comunidades, grupos ecológicos

\begin{abstract}
(Regeneration of tree species influenced by Merostachys multiramea Hack. (Poaceae) in a subtropical forest). The occurrence of bamboo species is common in several forest physiognomies, and the high abundance of these species may affect the regeneration of other ones. We evaluated both tree species and Merostachys multiramea Hack. recruitment in a transition area between mixed Araucaria rain forest and seasonal semideciduous forests in southern Brazil. We compared the regeneration structure of the areas evaluated two and four years after flowering, fruiting and death of M. multiramea. We randomly distributed $25100 \mathrm{~m}^{2}$ sampling units, and surveyed all living culms of M. multiramea and tree saplings. We sampled 4,372 living bamboos and 2,918 tree saplings, belonging to 32 plant families and 75 species. Most species were classified as zoochorous and canopy light-demanding. On the other hand, plant abundance revealed a greater contribution of autochorous, understory and shade-tolerant trees. M. multiramea density was higher over the four years after the reproductive event $(\mathrm{p}<0.05)$, with taller culms $(\mathrm{p}<0.01)$, but with the same mean number of culms ( $\mathrm{p}=0.46$ ). A NPMANOVA revealed significant differences in composition and abundance between surveys, with higher similarity among samplings two years after the reproductive event of $M$. multiramea. We also detected higher species richness over the study period, which was found to be directly associated with the abundance of $M$. multiramea ( $\mathrm{p}=0.002)$, but with lower evenness of abundance among regenerating species.
\end{abstract}

Key words: community ecology, ecological groups, patch dynamics, richness and diversity estimators

\footnotetext{
Universidade Regional Integrada do Alto Uruguai e das Missões, Laboratório de Sistemática e Ecologia Vegetal, Erechim, RS, Brasil

Universidade Federal de São Carlos, Programa de Pós-Graduação em Ecologia e Recursos Naturais, São Carlos, SP, Brasil

3 Autor para correspondência: jean@uricer.edu.br
} 


\section{Introdução}

A dinâmica florestal é frequentemente associada a processos organizacionais e de interações com variáveis ambientais, refletindo em padrões estruturais distintos ao longo do tempo e do espaço. Processos locais de morte e recrutamento de indivíduos estão da mesma forma associados às próprias interações entre espécies vegetais, que podem gerar facilitação ou competição entre indivíduos (Michalet et al. 2006). Dentre os exemplos mais comuns estão a relação entre plântulas e espécies berçário (Valient-Banuet et al. 2006) e de competição entre regeneração de espécies arbóreas e pioneiras de rápido crescimento, como as bambúseas (Campanello et al. 2007).

Em se tratando de processos dinâmicos em pequena escala, a formação de clareiras é um dos processos mais importantes na renovação florestal, uma vez que influencia de forma diferenciada as taxas de germinação, mortalidade e desenvolvimento de espécies florestais (Whitmore 1989). Em se tratando de espécies pioneiras e não-pioneiras, vários trabalhos têm demonstrado a estreita relação entre, por exemplo, níveis de luminosidade e a ocorrência de espécies associadas com suas respectivas necessidades ecológicas para esta variável (Clark et al. 1996).

Os bambus exercem um papel fundamental na dinâmica de florestas (Marchesini et al. 2009). A forma de reprodução vegetativa por meio de rizomas, o rápido crescimento e a elevada densidade de colmos por área tornam as espécies de bambu altamente competitivas. Alguns estudos realizados em florestas subtropicais e temperadas têm demonstrado que bambus são colonizadores agressivos e podem interferir na regeneração natural ao suprimir ou retardar o recrutamento e a colonização de espécies arbóreas, além de afetar a sobrevivência e o crescimento de indivíduos adultos (Gonzáles et al. 2002). Em áreas tropicais, a ocorrência de florestas dominadas por bambus foi associada à elevada mortalidade de plântulas na presença de bambúseas e a distúrbios ocasionados por ventos e propriedades mecânicas do solo (Griscom \& Ashton 2003), bem como a possíveis eventos catastróficos, como queimadas, as quais poderiam estar relacionadas a secas coincidentes com épocas de mortalidade de espécies monocárpicas (Nelson 1994).

Uma característica particular da maioria das espécies de bambu é o seu incomum ciclo de vida, que varia de 3 a 120 anos (McClure 1973), acabando com um único evento reprodutivo que leva à floração em massa e produção de sementes (McClure 1973; Keeley \& Bond 1999). Geralmente, esses episódios de floração sincronizada envolvem uma grande parte da população, embora ocasionalmente fragmentos de ramos não florescidos permaneçam. A produção de sementes durante eventos de floração é abundante; por exemplo, Guilherme \& Ressel (2001) calcularam a produção de sementes de Merotachys riedeliana em florestas sazonais no sudeste do Brasil em mais de 30 milhões de sementes.ha ${ }^{-1}$, e González et al. (2002) estimaram uma entrada de 195 milhões de sementes.ha ${ }^{-1}$ durante a floração de Chusquea quila em uma floresta de faias no sul do Chile. Este evento incomum gera outro episódio marcante destas áreas, que é o brusco aumento nas populações de granívoros, conhecidas como "ratadas" (Jansen 1976; Gallardo \& Mercado 1999; Jaksic e Lima 2003). Eventos de floração em bambus têm sido registrados há séculos, especialmente na Ásia e América (Taylor et al. 2004), mas os efeitos destes processos sobre a regeneração de espécies arbóreas ainda são pouco compreendidos, especialmente pelo longo tempo entre florações.

Merostachys multiramea Hack. é uma espécie de bambu de floração cíclica e que apresenta floração massiva a cada 31-33 anos (Schmitt \& Longhi-Wagner 2009), com o último evento reprodutivo registrado no sul do Brasil entre 2006 e 2008. Após a floração, todos os colmos de bambu morreram e geraram várias clareiras de tamanhos diferentes no interior das florestas (Budke et al. 2010).

O presente estudo faz parte de um projeto que visa identificar os processos e padrões associados à dinâmica de Merostachys multiramea Hack. em áreas do Brasil subtropical. Esta iniciativa teve início em 2008 (dois anos após floração e frutificação da bambúsea), quando as unidades amostrais utilizadas neste estudo foram demarcadas e os indivíduos arbóreos em regeneração e os de M. multiramea foram mensurados por Budke et al. (2010). Com a morte dos indivíduos, também se detectou aumento da temperatura do ar e menores níveis de umidade nas unidades amostrais onde havia maior quantidade de colmos. A regeneração de espécies arbóreas esteve altamente associada com a dinâmica do bambu, sendo que em áreas de dossel bem estruturado, houve menor número de espécies e menor diversidade específica. Naquele momento, entretanto, não foram verificadas diferenças entre a densidade de bambus, número de colmos por plântula e altura média dos colmos entre áreas distintas de alta e baixa densidade de bambu (Budke et al. 2010).

Nesta etapa, quatro anos após o término da floração e frutificação de M. multiramea, as unidades amostrais foram reavaliadas quanto aos mesmos parâmetros estruturais, possibilitando uma análise temporal comparativa entre levantamentos, destacando-se comparações entre grupos ecológicos de dispersão, necessidades de luz para germinação e tamanho médio dos indivíduos, bem como, uma avaliação da riqueza específica e da similaridade estrutural das áreas ao longo do tempo. Nossa hipótese inicial de trabalho foi de que pelo rápido crescimento vegetativo atribuído à $M$. multiramea e posterior competição mecânica com indivíduos arbóreos em regeneração, a riqueza específica tenha diminuído nas áreas com maior densidade de M. multiramea, bem como, a uniformidade entre as distribuições de abundância das espécies também deverá ter sido menor. 


\section{Material e métodos}

\section{Caracterização da área de estudo}

$\mathrm{O}$ estudo foi conduzido em um remanescente florestal pertencente ao Horto Florestal Municipal de Erechim, localizado próximo à rodovia RS-135, a $768 \mathrm{~m}$ de altitude média acima do nível do mar, na região Norte do Rio Grande do Sul, entre as coordenadas $27^{\circ} 42^{\prime} 41^{\prime \prime} \mathrm{e} 27^{\circ} 43^{\prime} 15^{\prime \prime}$ de latitude sul e $52^{\circ} 18^{\prime} 48^{\prime \prime}$ e $52^{\circ} 17^{\prime} 53^{\prime \prime}$ de longitude oeste. Historicamente, o clima da região foi descrito como Cfa, de acordo com a classificação climática de Köppen (Nimer 1990). Dados referentes à Estação Meteorológica de Erechim, instalada a $750 \mathrm{~m}$ acima do nível do mar, para o período de 1976 a 2005, apresentaram temperatura média anual de $17,6^{\circ} \mathrm{C}$, sendo a média mínima registrada para o mês de junho $\left(12,7^{\circ} \mathrm{C}\right)$ e a média máxima para o mês de janeiro $\left(25,5^{\circ} \mathrm{C}\right)$. As chuvas são bem distribuídas ao longo do ano e atingem precipitação média de 1912,3 mm.ano ${ }^{-1}$ (Bernardi \& Budke 2010).

O Horto Florestal é considerado uma unidade de proteção ambiental de categoria municipal, com área aproximada de 60 hectares. Caracteriza-se por incluir um remanescente de transição entre Floresta Ombrófila Mista e Floresta Estacional Semidecídua, inserido em uma matriz predominantemente agrícola, tendo em sua área de influência indireta propriedades caracterizadas pela agricultura familiar (Bernardi \& Budke 2010). Pelo mapeamento do solo realizado no estado do Rio Grande do Sul (Streck et al. 2008), o solo da área em estudo é classificado como sendo Latossolo Vermelho Aluminoférrico.

\section{Desenho amostral e coleta dos dados}

As coletas foram realizadas no período de dezembro de 2009 a fevereiro de 2010, em 25 unidades amostrais de $10 \mathrm{x}$ 10 metros alocadas por Budke et al. (2010) sendo dispostas de forma aleatória, em áreas onde inicialmente foi constatada a presença de morte de M. multiramea (12 unidades amostrais) e sem ocorrência de populações adultas desta bambúsea (13 unidades amostrais).

Em cada unidade amostral foram amostrados todos os indivíduos vivos de Merostachys multiramea Hack., quantificando-se o número de colmos existentes em cada touceira, o perímetro à altura do solo (PAS) de cada colmo e a altura média dos colmos. Para as plântulas de espécies arbóreas foram amostrados todos os indivíduos com altura mínima de $30 \mathrm{~cm}$ e até $15 \mathrm{~cm}$ de perímetro à altura do peito. Os indivíduos que se encontravam sobre a linha da extremidade das unidades amostrais foram considerados quando metade ou mais do indivíduo se encontrava presente dentro da unidade amostral. As espécies arbóreas foram identificadas in loco, ou então, foi coletado material botânico para posterior identificação no Laboratório de Sistemática e Ecologia Vegetal - ECOSSIS da Universidade Regional Integrada do Alto Uruguai e das Missões - Campus de Erechim. A nomenclatura das espécies seguiu Sobral et al. (2006).

\section{Análise dos dados}

Foram estimados o número médio de indivíduos de $M$. multiramea por área, o número médio de colmos e a altura média dos colmos por indivíduo. Da mesma forma foram calculados os parâmetros de densidade e freqüência das espécies arbóreas em regeneração, bem como o índice de valor de importância. As espécies foram distribuídas de acordo com as estratégias de dispersão, sendo divididas em zoocóricas, anemocóricas e autocóricas, conforme a morfologia dos frutos e/ ou sementes (van der Pijl 1982); quanto à estratificação, foram divididos em indivíduos do sub-bosque, indivíduos do dossel e indivíduos emergentes (Oliveira-Filho et al. 1994); quanto às necessidades de luz para germinação, foram divididas em espécies tolerantes à sombra, espécies dependentes de luz e espécies pioneiras (Swaine \& Whitmore 1988). Estes diferentes grupos ecológicos foram comparados entre si por testes de qui-quadrado, tanto para número de espécies observadas quanto para número de indivíduos (Zar 1996). Da mesma forma, foi aplicado um teste de qui-quadrado para se avaliar se ocorreram ou não variações na proporção destes grupos ecológicos aos dois e quatro anos após floração, frutificação e morte de M. multiramea.

A composição e distribuição de abundância das espécies ao longo dos levantamentos foram comparadas por meio de análise de variância multivariada - NPMANOVA (Andersson 2001), calculada com o índice de Bray-Curtis como medida de dissimilaridade entre unidades amostrais, dentro e entre levantamentos. Em seguida, foi realizada uma Análise de Coordenadas Principais - PCoA, de forma a comparar visualmente a similaridade entre os dois levantamentos.

Finalmente, foi avaliada a riqueza específica dos levantamentos por meio de curvas de rarefação, as quais geraram estimativas de riqueza de espécies a um valor padronizado de indivíduos amostrados, provendo desta forma, uma base estatística de comparação (Gotelli \& Colwell 2001). A relação existente entre densidade de plântulas de espécies arbóreas e densidade de M. multiramea foi avaliada por meio de regressão linear. Os resíduos desta análise foram então utilizados numa regressão linear tendo a riqueza de espécies observadas como variável dependente. Para comparação dos dados referente à regeneração dos indivíduos arbóreos entre levantamentos, foi utilizado o critério de inclusão de indivíduos entre 0,30 até 1 metro de altura. Todas as análises foram feitas com auxílio do programa PAST (Hammer \& Harper 2009).

\section{Resultados}

Após o massivo processo de florescimento, frutificação e posterior morte de M. multiramea, várias clareiras de tamanhos distintos foram criadas, muitas das quais formando bordas nítidas com o dossel contínuo adjacente. Foi amostrado um total de 2.918 indivíduos arbóreos em regeneração ( $30 \mathrm{~cm}$ de altura até $15 \mathrm{~cm}$ de PAP) pertencentes 
a 32 famílias e 75 espécies, com densidade total estimada em 11.672 ind.ha $^{-1}$. As famílias que apresentaram maior riqueza de espécies foram Fabaceae, com 11 espécies amostradas (34\% do total), e Myrtaceae, com dez espécies amostradas (31\% do total), seguidas por Lauraceae, com cinco espécies (16\% do total) (Tab. 1). Essas três famílias detiveram $81 \%$ das espécies amostradas. O maior valor de importância foi atribuído para a espécie Gymnanthes concolor, seguida por Cupania vernalis, Pilocarpus pennatifolius e Trichilia elegans.

A densidade comparativa média de plântulas em regeneração variou de $4.752 \pm 2.572$ (dois anos) para $5.784 \pm$ 1.920 após quatro anos do período reprodutivo (Tab. 2). A densidade média de $M$. multiramea aumentou de $7.388 \pm$ 4.609 (dois anos) para $17.488 \pm 21.459$ após quatro anos. Da mesma forma, houve incremento na altura média dos colmos, sem acréscimo significativo no número médio de colmos por indivíduo (Tab. 2).

Quanto aos grupos ecológicos de dispersão, necessidade de luz para germinação e estratificação, foi observada predominância de espécies zoocóricas (70\%), dependentes de luz (59\%) e formadoras de dossel (66\%) (Tab 3). Num outro extremo, houve baixa contribuição de espécies autocóricas $(8 \%)$, pioneiras (10\%) e de porte emergente (9\%). Entretanto, quando estas mesmas categorias foram analisadas quanto ao número de indivíduos amostrados, os resultados diferiram consideravelmente, havendo maior contribuição de indivíduos autocóricos (51\%) e zoocóricos (43\%), tolerantes à sombra (68\%) e de sub-bosque (70\%) (Tab. 3), ao passo que indivíduos anemocóricos (6\%), pioneiros (2\%) e formadores de dossel (16\%) ou emergentes (14\%) tiveram proporções consideravelmente menores. A comparação da participação das espécies nestas categorias ecológicas, ao longo de dois e quatro anos após o período reprodutivo de M. multiramea revelou pouca variação na contribuição de cada categoria, sem diferenças significativas (Tab. 4).

A partir da NPMANOVA, $(\mathrm{F}=2,3 ; \mathrm{p}=0,03)$ pode-se constatar que houve diferença significativa entre a composição de espécies e respectivas abundâncias entre os levantamentos, gerando estruturas fitossociológicas distintas ao longo do tempo. A Análise de Coordenadas Principais (Fig. 2) revelou a ocorrência de maior similaridade entre as unidades amostrais de dois anos e maior dispersão (menor similaridade) entre unidades amostrais de quatro anos. Os dois primeiros eixos de ordenação explicaram respectivamente $28,4 \%$ e $12,4 \%$ da inércia total, indicando cerca de $41 \%$ da variação total da matriz. Observou-se no primeiro eixo de ordenação um gradiente formado por unidades amostrais de ambos os levantamentos, indicando sobreposição de unidades (Fig. 1).

Foi verificada maior riqueza de espécies no levantamento feito quatro anos após a reprodução de M. multiramea, com diferenças significativas detectadas pelas curvas de rarefação (Fig. 2), a partir de 850 a 900 indivíduos amostrados para cada área. Não houve relação linear significativa entre a densidade de plântulas e indivíduos de M. multiramea $\left(\mathrm{R}^{2}=\right.$
0,04; $\mathrm{p}=0,31)$ (Fig. 3A), mas a análise de resíduos a posteriori revelou haver relação linear significativa com a riqueza estimada de espécies (EstS) $\left(\mathrm{R}^{2}=0,34 ; \mathrm{p}=0,002\right)$ (Fig. 3B). A diversidade específica, obtida com um estimador de uniformidade ( $H^{\prime}$ de Shannon) revelou maior equilíbrio entre as distribuições de abundâncias das espécies no primeiro levantamento ( $\mathrm{p}=0,002)$ (Tab. 4), embora se tenha registrado menor número de espécies naquele momento.

\section{Discussão}

Eventos documentados para espécies monocárpicas geralmente registram os processos de grande mudança estrutural que ocorrem no período pós-distúrbio, dentre os quais o aumento de radiação luminosa direta, aumento da temperatura média do ar e diminuição da umidade relativa do ar (Budke et al. 2010; Holz \& Veblen 2006), e as conseqüências destas alterações sobre a dinâmica de regeneração de espécies arbóreas (González et al. 2002). Em se tratando da dispersão de bambúseas, o processo deve envolver o estabelecimento de indivíduos em áreas de dossel parcialmente abertas, ou seja, oriundas de distúrbios em pequena escala. Por outro lado, após seu período de reprodução e geração direta de clareiras, estas populações poderiam recolonizar tal área, ou poderia ocorrer um processo de colonização por espécies arbóreas, segundo o modelo proposto por Griscom \& Ashton (2003). Por outro lado, Nelson (1994) afirmou que o estabelecimento e manutenção de populações de Guadua no sudoeste da Amazônia poderia ser em decorrência de eventos catastróficos de grande escala, ocasionados principalmente pelo fogo em associação a períodos de secas prolongadas e floração das bambúseas.

$\mathrm{Na}$ avaliação realizada dois anos após o período reprodutivo, observou-se que o número de indivíduos de $M$. multiramea foi semelhante entre áreas de dossel contínuo e clareiras recém formadas. Logo, verificou-se que a germinação e o estabelecimento de indivíduos ocorreram tanto em área aberta quanto em área de dossel contínuo, onde as variáveis ambientais analisadas, sobretudo níveis de radiação luminosa, foram significativamente menores (Budke et al. 2010). Aos quatro anos, com o desenvolvimento do presente estudo, verificou-se que embora a densidade de plântulas em regeneração não tenha se alterado significativamente, ocorreu um aumento de mais de três vezes na densidade de indivíduos de M. multiramea, além do aumento significativo na altura média dos colmos. Desta forma, torna-se evidente o rápido avanço desta espécie sobre as áreas anteriormente por ela ocupadas, não ocorrendo o seu desaparecimento, como poderia ser previsto em alguns modelos de exclusão competitiva (Griscom \& Ashton 2003). Tampouco, pode-se sustentar que a manutenção das populações tenha associação com eventos em grande escala.

Como as sementes de M. multiramea não apresentam dormência, é provável que a espécie já tenha alcançado seu pico de germinação, com densidade média de aproxi- 
Tabela 1. Famílias, espécies e parâmetros estruturais da regeneração arbórea amostrada em uma floresta subtropical no sul do Brasil (CE = grupo ecológico, sendo $\mathrm{Z}$ = zoocórica, $\mathrm{An}=$ anemocórica, $\mathrm{Au}=$ autocórica; $\mathrm{D}=$ dossel, $\mathrm{E}=$ emergente, $\mathrm{Sb}=$ sub-bosque; $\mathrm{Dl}=$ dependente de luz; $\mathrm{Ts}=$ tolerante à sombra, $\mathrm{P}=$ pioneira; $\mathrm{DA}$ = dominância absoluta, $\mathrm{FA}=$ freqüência absoluta e VI = valor de importância).

\begin{tabular}{|c|c|c|c|c|}
\hline FAMÍLIAS/Espécies & $\mathrm{CE}$ & $\mathrm{DA}$ & FA & VI \\
\hline \multicolumn{5}{|l|}{ ADOXACEAE } \\
\hline Sambucus australis Cham. \& Schltdl. & Z-D-Dl & 2 & 1 & 0,36 \\
\hline \multicolumn{5}{|l|}{ ANNONACEAE } \\
\hline Annona neosalicifolia $\mathrm{H}$. Rainer & Z-D-Dl & 6 & 3 & 1,04 \\
\hline \multicolumn{5}{|l|}{ APOCYNACEAE } \\
\hline Aspidosperma australe Müll.Arg. & An-D-Dl & 12 & 6 & 2,25 \\
\hline \multicolumn{5}{|l|}{ AQUIFOLIACEAE } \\
\hline Ilex brevicuspis Reissek & Z-D-Dl & 1 & 1 & 0,28 \\
\hline \multicolumn{5}{|l|}{ BORAGINACEAE } \\
\hline Cordia ecalyculata Vell. & Z-D-Dl & 2 & 1 & 0,45 \\
\hline Cordia trichotoma (Vell.) Arráb. ex Steud. & Z-D-Dl & 1 & 1 & 0,26 \\
\hline \multicolumn{5}{|l|}{ CANNABACEAE } \\
\hline Celtis iguanaea (Jacq.) Sarg. & Z-D-Dl & 2 & 1 & 0,40 \\
\hline \multicolumn{5}{|l|}{ CARDIOPTERIDACEAE } \\
\hline Citronella paniculata (Mart.) R.A. Howard & Z-D-Dl & 3 & 2 & 0,70 \\
\hline \multicolumn{5}{|l|}{ CELASTRACEAE } \\
\hline Maytenus aquifolia Mart. & Z-D-Ts & 2 & 1 & 0,33 \\
\hline \multicolumn{5}{|l|}{ ELAEOCARPACEAE } \\
\hline Sloanea monosperma Vell. & Z-D-Dl & 7 & 5 & 1,59 \\
\hline \multicolumn{5}{|l|}{ EUPHORBIACEAE } \\
\hline Gymnanthes concolor Spreng. & $\mathrm{Au}-\mathrm{Sb}-\mathrm{Ts}$ & 1429 & 25 & 115,88 \\
\hline Sapium glandulosum (L.) Morong & An-D-P & 7 & 3 & 0,96 \\
\hline Sebastiania brasiliensis Spreng. & Au-D-Dl & 32 & 13 & 5,92 \\
\hline Sebastiania commersoniana (Baill.) L.B. Sm. \& Downs & $\mathrm{Au}-\mathrm{D}-\mathrm{P}$ & 11 & 5 & 2,13 \\
\hline \multicolumn{5}{|l|}{ FABACEAE } \\
\hline Albizia edwallii (Hoehne) Barneby \& J. Grimes & An-D-Dl & 13 & 7 & 2,15 \\
\hline Apuleia leiocarpa (Vogel) J.F. Macbr. & An-E-Dl & 5 & 1 & 0,40 \\
\hline Calliandra foliolosa Benth. & $\mathrm{Au}-\mathrm{D}-\mathrm{Ts}$ & 6 & 3 & 1,08 \\
\hline Dalbergia frutescens (Vell.) Britton & An-D-Dl & 23 & 11 & 3,65 \\
\hline Enterolobium contortisiliquum (Vell.) Morong & Au-D-Dl & 1 & 1 & 0,27 \\
\hline Inga marginata Willd. & Z-Sb-P & 18 & 10 & 3,58 \\
\hline Lonchocarpus campestris Mart. ex. Benth. & An-D-Dl & 37 & 14 & 5,3 \\
\hline Lonchocarpus nitidus (Vogel) Benth. & An-D-Dl & 31 & 14 & 5,48 \\
\hline Machaerium paraguariense Hassl. & An-D-Dl & 4 & 4 & 1,46 \\
\hline Myrocarpus frondosus Allemão & An-D-Dl & 2 & 2 & 0,53 \\
\hline Parapiptadenia rigida (Benth.) Brenan & An-E-Dl & 15 & 9 & 2,71 \\
\hline \multicolumn{5}{|l|}{ LAURACEAE } \\
\hline Aiouea saligna Meisn. & Z-D-Ts & 2 & 1 & 0,29 \\
\hline Endlicheria paniculata (Spreng.) J.F. Macbr. & Z-D-Ts & 6 & 1 & 0,44 \\
\hline
\end{tabular}


Tabela 1. Continuação.

\begin{tabular}{|c|c|c|c|c|}
\hline FAMÍLIAS/Espécies & $\mathrm{CE}$ & DA & FA & IVI \\
\hline Nectandra lanceolata Ness & Z-E-Dl & 21 & 4 & 1,77 \\
\hline Nectandra megapotamica (Spreng.) Mez & Z-D-Dl & 80 & 19 & 7,87 \\
\hline Ocotea diospyrifolia (Meisn.) Mez & Z-D-Ts & 8 & 5 & 1,73 \\
\hline \multicolumn{5}{|l|}{ MALVACEAE } \\
\hline Luehea divaricata Mart. \& Zucc. & An-D-P & 3 & 3 & 0,80 \\
\hline \multicolumn{5}{|l|}{ MELASTOMATACEAE } \\
\hline Miconia cinerascens Miq. & Z-Sb-Ts & 4 & 2 & 0,59 \\
\hline \multicolumn{5}{|l|}{ MELIACEAE } \\
\hline Cedrela fissilis Vell. & An-E-Dl & 7 & 3 & 1,08 \\
\hline Trichilia clausseni C.DC. & Z-Sb-Ts & 76 & 19 & 8,63 \\
\hline Trichilia elegans A. Juss. & Z-Sb-Ts & 104 & 21 & 12,08 \\
\hline \multicolumn{5}{|l|}{ MONIMIACEAE } \\
\hline Hennecartia omphalandra J. Poiss. & Z-Sb-Ts & 14 & 8 & 3,04 \\
\hline \multicolumn{5}{|l|}{ PRIMULACEAE } \\
\hline Myrsine loefgrenii (Mez) Imkhan & Z-Sb-Dl & 6 & 5 & 1,40 \\
\hline Myrsine umbellata Mart. & Z-D-Dl & 14 & 9 & 2,79 \\
\hline \multicolumn{5}{|l|}{ MYRTACEAE } \\
\hline Campomanesia xanthocarpa O.Berg & Z-D-Ts & 6 & 4 & 1,16 \\
\hline Eugenia involucrata DC. & Z-D-Dl & 6 & 4 & 1,21 \\
\hline Eugenia moraviana O.Berg & Z-D-Ts & 1 & 1 & 0,38 \\
\hline Eugenia pyriformis Cambess. & Z-D-Ts & 4 & 4 & 1,13 \\
\hline Eugenia rotundicosta D. Legrand & Z-D-Ts & 7 & 5 & 1,65 \\
\hline Eugenia subterminalis DC. & Z-D-Ts & 3 & 1 & 0,33 \\
\hline Eugenia uniflora $\mathrm{L}$. & Z-D-Dl & 3 & 2 & 0,61 \\
\hline Myrceugenia miersiana (Gardner) D. Legrand \& Kausel & Z-D-Dl & 1 & 1 & 0,33 \\
\hline Myrcia hebepetala DC. & Z-D-Ts & 10 & 6 & 1,91 \\
\hline Myrcia selloi (Spreng.) N. Silveira & Z-D-Dl & 7 & 5 & 1,52 \\
\hline \multicolumn{5}{|l|}{ NYCTAGINACEAE } \\
\hline Pisonia zapallo Griseb & Z-Sb-Ts & 1 & 1 & 0,29 \\
\hline \multicolumn{5}{|l|}{ PHYTOLACCACEAE } \\
\hline Seguieria aculeata Jacq. & An- D-Dl & 6 & 6 & 1,6 \\
\hline \multicolumn{5}{|l|}{ PICRAMINIACEAE } \\
\hline Picramnia sellowii Planch. & Z-D-Dl & 3 & 3 & 0,81 \\
\hline \multicolumn{5}{|l|}{ PIPERACEAE } \\
\hline Piper aduncum $\mathrm{L}$. & Z-Sb-Ts & 6 & 4 & 1,20 \\
\hline \multicolumn{5}{|l|}{ POLYGONACEAE } \\
\hline Ruprechtia laxiflora Meisn. & An-D-Dl & 2 & 2 & 0,54 \\
\hline RHAMNACEAE & & & & \\
\hline
\end{tabular}


Tabela 1. Continuação.

\begin{tabular}{|c|c|c|c|c|}
\hline FAMÍLIAS/Espécies & $\mathrm{CE}$ & DA & $\mathrm{FA}$ & IVI \\
\hline Hovenia dulcis Thunb. & Z-D-P & 1 & 1 & 0,40 \\
\hline \multicolumn{5}{|l|}{ RUBIACEAE } \\
\hline Cordiera concolor (Cham.) Kuntze. & Z-Sb-Ts & 2 & 2 & 0,65 \\
\hline Coussarea contracta (Walp.) Müll.Arg. & $\mathrm{Z}-\mathrm{Sb}$-Ts & 17 & 11 & 3,37 \\
\hline Rudgea parquioides (Cham.) Müll.Arg. & $\mathrm{Z}-\mathrm{Sb}$-Ts & 7 & 6 & 1,90 \\
\hline \multicolumn{5}{|l|}{ RUTACEAE } \\
\hline Balfourodendron riedelianum (Engl.) Engl. & An-E-Dl & 10 & 6 & 1,75 \\
\hline Pilocarpus pennatifolius Lem. & $\mathrm{Z}-\mathrm{Sb}-\mathrm{Ts}$ & 268 & 24 & 24,91 \\
\hline Zanthoxylum petiolare A. St.-Hil \& Tul. & Z-D-Dl & 2 & 1 & 0,30 \\
\hline Zanthoxylum rhoifolium Lam. & Z-D-Dl & 2 & 2 & 0,52 \\
\hline \multicolumn{5}{|l|}{ SALICACEAE } \\
\hline Banara tomentosa Clos. & Z-D-Dl & 3 & 2 & 0,56 \\
\hline Casearia decandra Jacq. & Z-D-Ts & 13 & 10 & 2,87 \\
\hline Casearia silvestris $\mathrm{Sw}$. & Z-D-Dl & 21 & 5 & 1,95 \\
\hline \multicolumn{5}{|l|}{ SAPINDACEAE } \\
\hline Allophylus edulis (A. St.-Hil., Cambess. \& A. Juss.) Radlk. & Z-D-Dl & 35 & 15 & 5,77 \\
\hline Cupania vernalis Cambess. & Z-E-Dl & 358 & 23 & 24,91 \\
\hline Matayba elaeagnoides Radlk. & Z-Sb-Dl & 32 & 12 & 4,07 \\
\hline \multicolumn{5}{|l|}{ SAPOTACEAE } \\
\hline Chrysophyllum gonocarpum (Mart. \& Eichler) Engl. & Z-Sb-Dl & 14 & 7 & 2,33 \\
\hline Chrysophyllum marginatum (Hook. \& Arn.) Radlk. & $\mathrm{Z}-\mathrm{Sb}-\mathrm{Dl}$ & 20 & 12 & 3,82 \\
\hline Pouteria gardneriana (DC.) Radlk. & Z-D-Dl & 3 & 3 & 0,83 \\
\hline \multicolumn{5}{|l|}{ SOLANACEAE } \\
\hline Cestrum bracteatum Link \& Otto & Z-Sb-Ts & 3 & 2 & 0,56 \\
\hline Cestrum strigilatum Ruiz \& Pav. & Z-Sb-P & 5 & 3 & 1,01 \\
\hline Solanum sanctaecatharinae Dunal & Z-D-Dl & 2 & 2 & 0,54 \\
\hline \multicolumn{5}{|l|}{ STYRACACEAE } \\
\hline Styrax leprosus Hook. \& Arn. & Z-D-Dl & 15 & 6 & 1,98 \\
\hline \multicolumn{5}{|l|}{ SYMPLOCACEAE } \\
\hline Symplocos lanceolata DC. & Z-D-Dl & 2 & 1 & 0,31 \\
\hline
\end{tabular}

Tabela 2. Comparação do número médio de indivíduos, número médio de colmos por indivíduo e altura média dos colmos de Merostachys multiramea Hack., avaliados dois e quatro anos após período reprodutivo em uma floresta subtropical no sul do Brasil. Valores representam a média \pm desvio padrão.

\begin{tabular}{lcr}
\hline & dois anos & quatro anos \\
\hline Densidade de plântulas (ind. ha ${ }^{-1}$ ) & $4.752 \pm 2.572$ & $5.784 \pm 1.920$ \\
Densidade de M. multiramea (ind. ha ${ }^{-1}$ ) & $7.388 \pm 4.609$ & $17.488 \pm 21.459$ \\
Colmos por indivíduo & $3,9 \pm 0,9$ & $3,8 \pm 0,5$ \\
Altura média dos colmos (m) & $0,8 \pm 0,4$ & $1,1 \pm 0,4$ \\
\hline
\end{tabular}


Tabela 3. Proporção de espécies e indivíduos em diferentes grupos ecológicos amostrados em uma floresta subtropical no sul do Brasil, quatro anos após período reprodutivo de Merostachys multiramea Hack. Testes de qui-quadrado $\left(\chi^{2}\right)$ foram aplicados comparando as diferentes categorias ecológicas para proporção de espécies e indivíduos em cada categoria.

\begin{tabular}{|c|c|c|c|c|}
\hline Categoria ecológica & $\%$ de Spp & $\chi^{2}$ & $\%$ de Ind & $x^{2}$ \\
\hline \multicolumn{5}{|l|}{ Estratégias de dispersão } \\
\hline Zoocóricas & 70 & & 43 & \\
\hline Anemocóricas & 22 & $63,44^{*}$ & 6 & $34,58^{*}$ \\
\hline Autocóricas & 8 & & 51 & \\
\hline \multicolumn{5}{|l|}{ Necessidades de luz } \\
\hline Tolerantes à Sombra & 31 & & 68 & \\
\hline Dependentes de Luz & 59 & $36,26^{*}$ & 30 & $65,84^{*}$ \\
\hline Pioneiras & 10 & & 2 & \\
\hline \multicolumn{5}{|l|}{ Estratificação/porte } \\
\hline Sub-bosque & 25 & & 70 & \\
\hline Dossel & 66 & $51,86^{*}$ & 16 & $60,56^{*}$ \\
\hline Emergente & 9 & & 14 & \\
\hline
\end{tabular}

* $=\mathrm{p}<0,001 ; n s=$ não significativo

madamente 17.500 plântulas.ha-1, valor muito menor que os relatados para espécies do gênero Chusquea, como no exemplo de González et al. (2002) onde o número de plântulas pode chegar nas primeiras fases de desenvolvimento a cerca de 360.000 ind. ha ${ }^{-1}$.

A maioria das espécies amostradas tem dispersão zoocórica, e é dependente de luz para germinação, além de caracterizarem-se como espécies de grande porte, ou seja, formadores de dossel. Houve baixa incidência de espécies pioneiras, as quais são geralmente abundantes em clareiras recentes. Por outro lado, houve maior contribuição das classes de autocoria, tolerantes à sombra e de sub-bosque, ao se observar o número de indivíduos amostrados. Este padrão já fora observado em outros trabalhos realizados no Brasil meridional (Giehl et al. 2007) e está provavelmente associado à alta proporção de indivíduos de Gymnanthes concolor, espécie de sub-bosque e com elevada densidade em florestas estacionais e florestas com Araucária (Jarenkow \& Budke 2009).

De acordo com os critérios de inclusão adotados neste estudo (mínimo de $30 \mathrm{~cm}$ de altura), é muito provável que grande parte dos indivíduos amostrados tenha se estabelecido antes do processo de morte de M. multiramea. Ou seja, a estrutura de regeneração ora observada é um reflexo de processos que ocorreram antes, durante e após a reprodução desta espécie, com mudanças estruturais em curto prazo. Estas fases, chamadas de "fase de reorganização" e "fase de novos recrutamentos" (sensu González et al. 2002), envolvem o crescimento acelerado das plântulas já existentes durante o evento, bem como o recrutamento a partir de sementes que chegaram nas clareiras ou que faziam parte do prévio banco de sementes. Esta premissa é reforçada pela análise de variância multivariada não-para- métrica, onde se verificou que a estrutura de regeneração entre as datas de amostragem variou significativamente, ou seja, ocorreu elevado recrutamento a partir do banco de sementes existente, bem como o crescimento dos indivíduos pré-existentes. Da mesma forma, a ordenação das unidades amostrais revelou maior dispersão entre as unidades do levantamento de quatros anos, indicando que ao longo da área, ocorrem processos que se constituem numa dinâmica de mosaicos.

De acordo com Chávez \& MacDonald (2010), o processo de dinâmica de mosaicos é comum em ambientes florestais, e pode refletir em mudanças ambientais oriundas de distúrbios recorrentes, como inundações ou formação de clareiras, ou então, em função dos diferentes conjuntos ou estandes que formam um remanescente florestal. Em se tratando do presente estudo, as curvas de rarefação indicaram maior riqueza estimada de espécies no levantamento de quatro anos após distúrbio, indicando condições favoráveis para o recrutamento de espécies arbóreas em uma ambiente com gradativo aumento de biomassa de $M$. multiramea. Da mesma forma, os resultados das análises de regressão revelaram a ausência de relação entre densidade da bambúsea e a densidade de plântulas de espécies arbóreas. O resíduo desta relação é, entretanto, um preditor linear positivo da riqueza específica. Com o decorrer do tempo, percebeu-se uma diminuição na uniformidade entre as distribuições de abundância das espécies, gerando estimativas de diversidade menores quatro anos após distúrbio, embora se tenha registrado um incremento na riqueza específica. Este processo pode ser decorrente da grande variação de abundância de algumas espécies ao longo do tempo, sobretudo de Gymnanthes concolor, a qual variou de 409 ind.ha-1 (Budke et al. 2010) para 1.429 ind.ha ${ }^{-1}$ após quatro anos, ocasionando um recrutamento 
Tabela 4. Comparação entre a proporção de espécies em diferentes grupos ecológicos e índice de diversidade de Shannon (H`) entre 2 anos (Budke et al 2010) e 4 anos (este estudo) após a floração, frutificação e morte de Merostachys multiramea Hack. em uma floresta subtropical no sul do Brasil.

\begin{tabular}{|c|c|c|c|}
\hline & 2 anos & 4 anos & $x^{2}$ \\
\hline \multicolumn{4}{|l|}{ Estratégia de dispersão } \\
\hline Zoocóricas & 75 & 70 & $0,17 \mathrm{~ns}$ \\
\hline Anemocóricas & 18 & 22 & $0,40 \mathrm{~ns}$ \\
\hline Autocóricas & 7 & 8 & $0,06 \mathrm{~ns}$ \\
\hline$x^{2}$ & $79,94^{*}$ & $63,44^{*}$ & $0,63 \mathrm{~ns}$ \\
\hline \multicolumn{4}{|l|}{ Necessidade de luz } \\
\hline Tolerante á sombra & 30 & 31 & $0,01 \mathrm{~ns}$ \\
\hline Dependente de luz & 63 & 59 & $0,13 \mathrm{~ns}$ \\
\hline Pioneira & 7 & 10 & $0,52 \mathrm{~ns}$ \\
\hline$x^{2}$ & $47,54^{\star}$ & $36,26^{*}$ & $0,67 \mathrm{~ns}$ \\
\hline \multicolumn{4}{|l|}{ Estratificação/ porte } \\
\hline Sub-bosque & 33 & 25 & $1,10 \mathrm{~ns}$ \\
\hline Dossel & 53 & 66 & $1,42 \mathrm{~ns}$ \\
\hline Emergente & 14 & 9 & $1,08 \mathrm{~ns}$ \\
\hline$x^{2}$ & $22,82^{*}$ & $51,86^{*}$ & $3,61 \mathrm{~ns}$ \\
\hline H’ Shannon & 2,595 nats.ind $^{-1}$ & 2,40 nats.ind $^{-1}$ & $p=0,02$ \\
\hline
\end{tabular}

${ }^{*}=\mathrm{p}<0,001 ; n s=$ não significativo

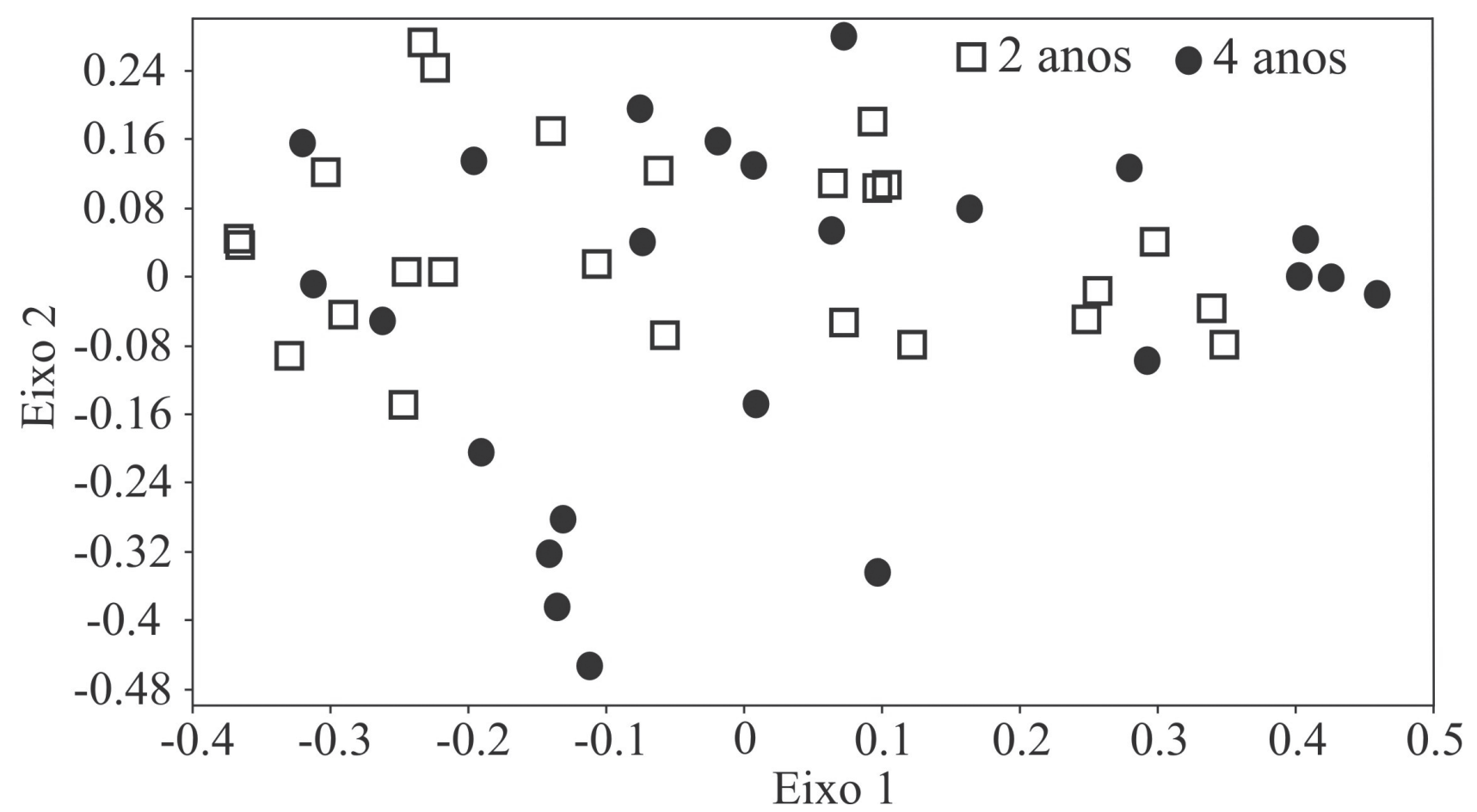

Figura 1. Análise de coordenadas principais ( $\mathrm{PCoA})$ entre unidades amostrais de levantamentos de regeneração arbórea realizados em dois e quatro anos após floração, frutificação e morte de Merostachys multiramea Hack. em uma floresta subtropical no sul do Brasil. 


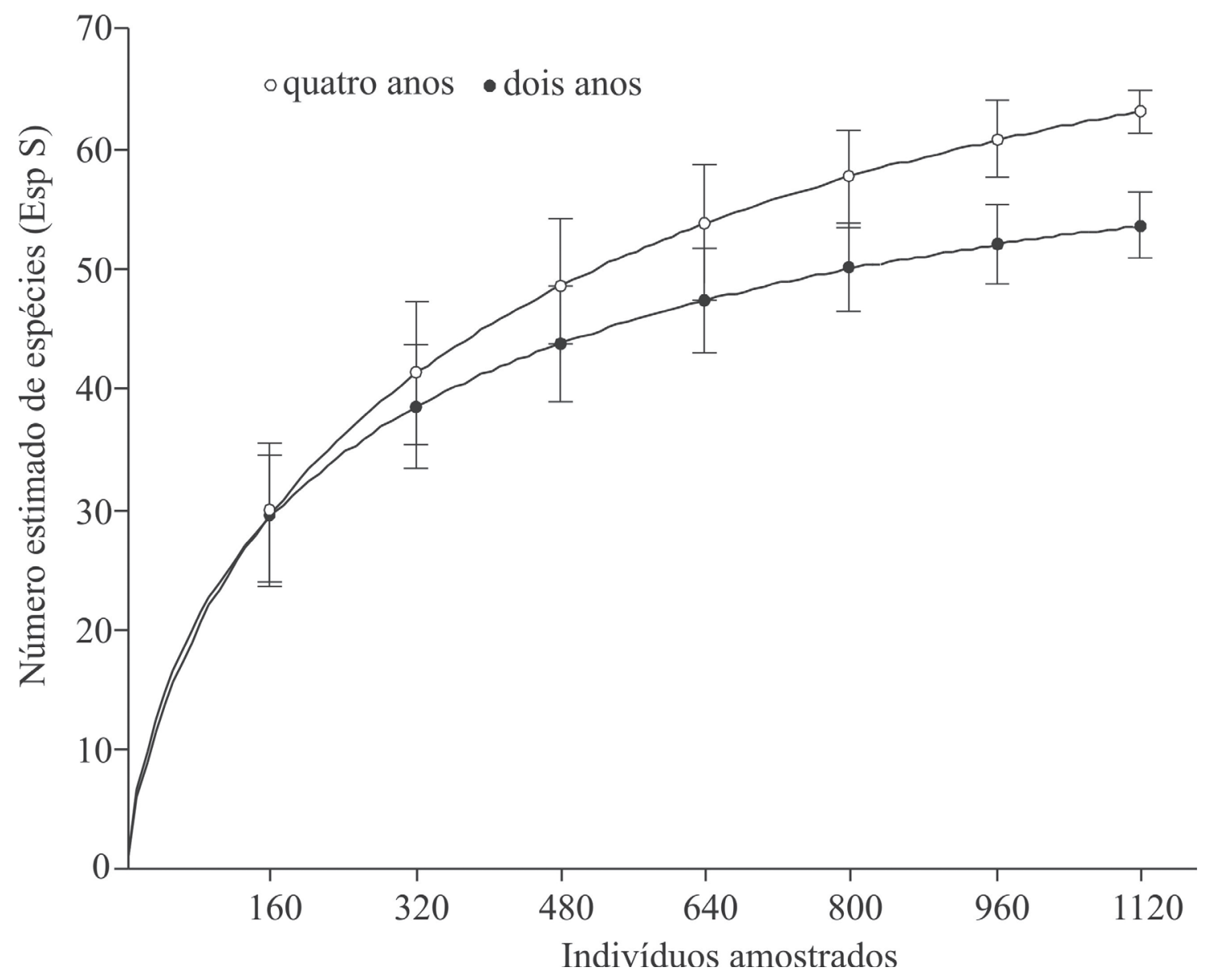

Figura 2. Curvas de rarefação de espécies entre unidades amostrais de levantamentos de regeneração arbórea realizados em dois e quatro anos após floração, frutificação e morte de Merostachys multiramea Hack. em uma floresta subtropical no sul do Brasil.
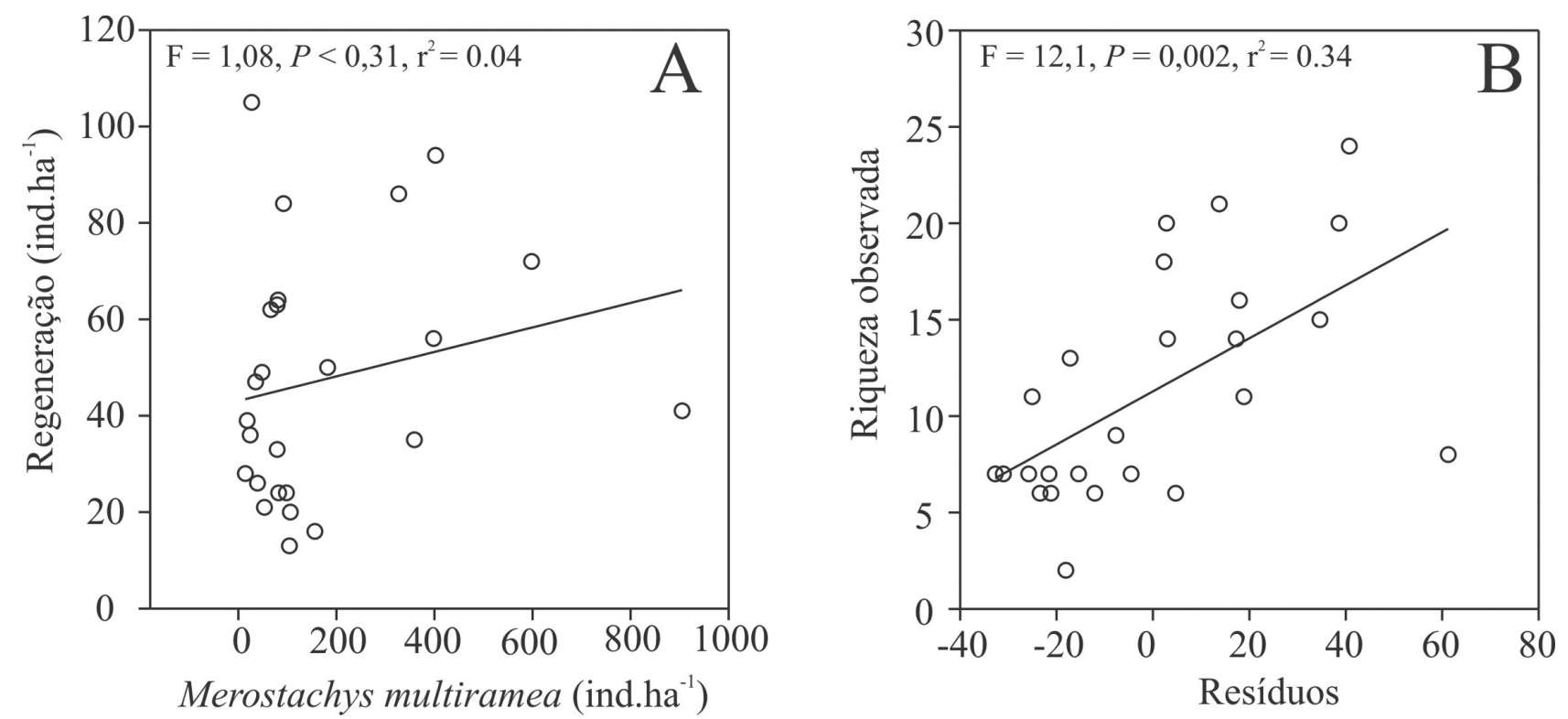

Figura 3. Análises de regressão entre densidade de Merostachys multiramea Hack. e densidade de plântulas em regeneração (A) e análise de regressão entre os respectivos resíduos e a riqueza observada de espécies entre unidades amostrais de levantamentos realizados quatro anos após floração, frutificação e morte de Merostachys multiramea Hack. em uma floresta subtropical no sul do Brasil. 
desigual para as espécies, sobretudo aquelas formadoras de banco de plântulas, como é o caso desta espécie (Giehl et al. 2007). Estes autores discutem ainda que Gymnanthes concolor suporta elevadas densidades, e que possui geralmente distribuição agrupada, resultado do mecanismo de dispersão autocórico, moldado por dispersão dos diásporos em curtas distâncias, com muitos indivíduos sendo recrutados próximos da planta mãe.

Conforme já discutido por Marchesini et al. (2009) e Budke et al. (2010), a abrupta germinação de sementes de $M$. multiramea provavelmente leva a espécie a não formar banco de sementes. Além disto, o aumento do número de indivíduos, bem como da altura média dos colmos reflete no vigor da espécie em ocupar tanto áreas de dossel contínuo, quanto áreas abertas pela morte e queda da população antiga (Budke et al. 2010). Este rápido estabelecimento gerou uma elevada densidade populacional, a qual poderá ser estudada ao longo do tempo, quanto à formação e manutenção de banco de plântulas. Caso esta hipótese se confirme, é provável que M. multiramea se mantenha no remanescente ao longo do tempo, confirmando a "hipótese de recolonização" sensu Griscom \& Ashton (2003). Neste caso, um maior estabelecimento de indivíduos arbóreos seria dependente da ocorrência de eventos sincrônicos e que dificultassem a germinação de sementes da bambúsea, especialmente em áreas com franca competição por espaço, onde, na maioria dos casos, a competição entre bambu e plântulas de espécies arbóreas gera injúrias ocasionadas pela carga de biomassa sobre galhos (Griscom \& Ashton 2006). Da mesma forma, estudos abrangendo o manejo de M. multiramea, especialmente quanto ao potencial de estabelecimento de espécies arbóreas, serão cruciais para se avaliar até que ponto a presença destas espécies pode interferir na dinâmica florestal. Até o presente momento, quatro anos após a floração, frutificação e morte das populações de M. multiramea, é notório que a riqueza de espécies aumentou ao longo do tempo, corroborando outros estudos com dinâmica de bambúseas no sul da América (Holz \& Veblen 2006; Rafaelle et al. 2007; Marchesini et al. 2009), sendo possível uma avaliação destas variáveis ao longo do tempo, sobretudo quanto à necessidade de manejo das áreas com elevada densidade desta bambúsea.

\section{Agradecimentos}

Ao Laboratório de Sistemática e Ecologia Vegetal ECOSSIS da Universidade Regional Integrada do Alto Uruguai e das Missões - URI, Campus de Erechim, pelo apoio financeiro e logístico. Os autores agradecem à Prefeitura Municipal de Erechim, por meio da Secretaria Municipal de Meio Ambiente, pela permissão de coleta e execução do trabalho junto ao Horto Florestal Municipal. Aos revisores anônimos, pelas sugestões e recomendações.

\section{Referências bibliográficas}

Anderson, M.J., 2001. A new method for non-parametric multivariate analysis of variance. Austral Ecology 26: 32-46.

Bernardi, S. \& Budke, J.C. 2010. Estrutura da sinúsia epifítica e efeito de borda em uma área de transição entre Floresta Estacional Semidecídua e Floresta Ombrófila Mista. Revista Floresta 40: 81-92.

Budke, J.C.; Alberti, M.S.; Zanardi, C.; Barrato, C. \& Zanin, E.M. 2010. Bamboo dieback and tree regeneration responses in a subtropical forest of South America. Forest Ecology and Management 260: 1345-1349.

Campanello, P.I.; Gatti, M.G.; Ares, A.; Montti, L. \& Goldstein, G., 2007. Tree regeneration and microclimate in a liana and bamboo-dominated semideciduous Atlantic forest. Forest Ecology and Management 252: 108-117.

Chávez, V. \& Macdonald, S.E. 2010. The influence of canopy patch mosaics on understory plant community composition in boreal mixedwood forest. Forest Ecology and Management 259: 1067-1075.

Clark, D.B.; Clark, D.A.; Rich, P.M.; Weiss, S. \& Oberbauer, S.F. 1996. Landscape-scale evaluation of understory light and canopy structure: methods and application in a neotropical lowland rain forest. Canadian Journal of Forest Research 26: 747-757.

Gallardo, M.H. \& Mercado, C.L. 1999. Mast seeding of bamboo shrubs and mouse outbreak in Southern Chile. Mastozoologia Neotropical 6: 103-111.

Giehl, E.L.H.; Athayde, E.A.; Budke, J.C.; Gesing, J.P.A.; Eisinger, S.M. \& Canto-Dorow, T.S. 2007. Espectro e distribuição vertical das estratégias de dispersão de diásporos do componente arbóreo em uma floresta estacional no sul do Brasil. Acta Botanica Brasilica 21: 137-145.

González, M.; Veblen, T.; Donoso, C. \& Valeria, L. 2002. Tree regeneration responses in a lowland Nothofagus-dominated forest after bamboo dieback in South- Central Chile. Plant Ecology 161: 59-73.

Gotelli, N.J. \& Colwell, R.K. 2001. Quantifying biodiversity: procedures and pitfalls in the measurement and comparison of species richness. Ecology Letters 4: 379-391.

Griscom, B.W. \& Ashton, M.S. 2003. Bamboo control of forest succession: Guadua sarcocarpa in southeastern Peru. Forest Ecology and Management 175: 445-454.

Griscom, B.W. \& Ashton, M.S. 2006. A self-perpetuating bamboo disturbance cycle in a neotropical forest. Journal of Tropical Ecology 22: 587-597.

Guilherme, F.A.G. \& Ressel, K. 2001. Biologia floral e sistema de reprodução de Merostachys riedeliana (Poaceae: Bambusoideae). Revista Brasileira de Botânica 24: 205-211.

Hammer, O. \& Harper, D.A.T. 2011. PAST version 2.06. Disponível em http://folk.uio.no/ohammer/past (Acesso em 31/01/2011).

Holz, C.A. \& Veblen, T., 2006. Tree regeneration responses to Chusquea montana bamboo die-off in a subalpine Nothofagus forest in the southern Andes. Journal of Vegetation Science 17: 19-28.

Jaksic, F.M. \& Lima, M. 2003. Myths and facts about ratadas: bamboo blooms, rainfall peaks and rodent outbreaks in South America. Austral Ecology 28: 237-251.

Janzen, D.H. 1976. Why bamboos wait so long to flower? Annual Review of Ecology \& Systematics 7: 347-391.

Jarenkow, J.A. \& Budke, J.C. 2009. Padrões florísticos e análise estrutural de remanescentes de Florestas com Araucária no Brasil. Pp. 113-126. In: Fonseca, C.R.; Souza, A.F.; Leal-Zanchet, A.M.; Dutra, T.; Backes, A. \& Ganade, G. (Org.). Floresta com araucária: ecologia, conservação e desenvolvimento sustentável. Ribeirão Preto, Holos Editora.

Keeley, J.E. \& Bond, W.J.1999. Mast flowering and semelparity in bamboos: the bamboo fire cycle hypothesis. American Naturalist 154: 383-391.

Marchesini, V.A.; Sala, O.E. \& Austin, A.T., 2009. Ecological consequences of a massive flowering event of bamboo (Chusquea culeou) in a temperate forest of Patagonia, Argentina. Journal of Vegetation Science 40: 424-432.

McClure, F.A. 1973. Genera of bamboos native to the New World (Gramineae: Bambusoideae). Smithsonian Contribution to Botany 9: 1-148.

Michalet, R.; Brooker, R.W.; Cavieres, L.A.; Kikvidze, Z.; Lortie, C.J.; Pugnaire, F.I.; Valiente-Banuet, A. \& Callaway, R.M. 2006. Do biotic 
interactions shape both sides of the humped-back model of species richness in plant communities? Ecology Letters 9: 767-773.

Nelson, B.W. 1994. Natural Forest disturbance and changes in the Brazilian Amazon. Remote Sensing Review 10: 105-125.

Nimer, E. 1990. Clima. Pp. 151-187. In: IBGE. Geografia do Brasil: região Sul. Rio de Janeiro, IBGE.

Oliveira-Filho, A.T.; Vilela, E.A.; Carvalho, D.A. \& Gavilanes M.L. 1994. Effects of soils and topography on the distribution of tree species in a tropical riverine forest in south-eastern Brazil. Journal of Tropical Ecology 10: 483-508.

Rafaelle, E.; Kitzberger, T. \& Veblen, T.T. 2007. Interactive effects of introduced herbivores and post-flowering die-off of bamboos in Patagonia Nothofagus forests. Journal of Vegetation Science 18: 371-378.

Schmidt, R. \& Longhi-Wagner, H.M. 2009. A tribo bambuseae (Poaceae: Bambusoideae) no Rio Grande do Sul. Brasil. Revista Brasileira de Biociências 7: 71-128.

Sobral, M.; Jarenkow, J.A.; Brack, P.; Irgang, B.; Larocca, J. \& Rodrigues, R.S. 2006. Flora arbórea e arborescente do Rio Grande do Sul, Brasil. São Carlos, RiMA/Novo Ambiente.
Streck, E.V.; Kampf, N.; Dalmolin, R.S.D.; Klamt, E.; do Nascimento, P.C.; Scheneider, P.; Giasson, E. \& Pinto, L.F.S. 2008. Solos do Rio Grande do Sul. 2 ed. Porto Alegre, EMATER-RS.

Swaine, M.D. \& Whitmore, T.C. 1988. On the definition of ecological species groups in tropical rain forests. Vegetatio 75: 81-86.

Taylor, A.H.; Jinyan, H. \& Zhou, S. 2004. Canopy tree development and undergrowth bamboo dynamics in old-growth Abies-Betula forest in southwestern China: a 12-year study. Forest Ecology and Management 200: 347-360.

Valiente-Banuet, A.; Rumebe, A.V.; Verdú, M. \& Callaway, R.M. (2006) Quaternary Plant lineages sustain global diversity by facilitating Tertiary lineages. Proceedings of the National Academy of Sciences 103: 16812-16817.

van der Pijl, L. 1982. Principles of dispersal in higher plants. New York, Springer Verlag.

Whitmore, T.C. 1989. Canopy gaps and the two major groups of forest trees. Ecology 70: 536-538.

Zar, J. H. 1996. Biostatistical analysis. New Jersey, Prentice-Hall. 